\title{
TRACKING CONTROL OF A FLEXIBLE BEAM BY NONLINEAR BOUNDARY FEEDBACK ${ }^{1}$
}

\author{
BAO-ZHU GUO \\ Beijing University of Technology \\ Department of Applied Mathematics \\ Beijing 100081, China \\ QIAN SONG \\ Academia Sinica \\ Institute of Systems Science \\ Beijing 100080, China
}

(Received February, 1994; revised November, 1994)

\begin{abstract}
This paper is concerned with tracking control of a dynamic model consisting of a flexible beam rotated by a motor in a horizontal plane at the one end and a tip body rigidly attached at the free end. The well-posedness of the closed loop systems considering the dissipative nonlinear boundary feedback is discussed and the asymptotic stability about difference energy of the hybrid system is also investigated.
\end{abstract}

Key words: Flexible Beam, Exponential Decay, Stability, Tracking Control, Boundary Feedback Control.

AMS (MOS) subject classifications: 93D15.

\section{Introduction and System Formulation}

Mechanical flexibility in motion control systems attracted more attention in recent years. Motivated by [4], in which a hybrid system describing the overhead crane model was studied, we will consider in this paper a flexible beam rotated by a motor in a horizontal plane at one end and a top body rigidly attached at the free end. This model fits a large class of real applications such as links of robot system and space-shuttle arms in which high speed manipulation and long and slender geometrical dimensions are the major factors causing mechanical vibration. To achieve high speed and precision end point positioning of the flexible beam (which must be guaranteed in any condition variations such as payload) the boundary control is one of the major strategies in production and space applications.

Let $\ell$ be the length of the beam, $\rho$ the uniform mass density per unit length, $E I$ the uniform flexural rigidity and $m$ be the mass of the tip body attached at the free end of the link, $I_{m}$ the moment of inertia of the motor and $J$ the moment of inertia associated with the tip body. Taking the motor's torque as the control input and neglecting rotary inertia and shear deformation effects and actuator dynamics, the total transversal displacement $y(x, t)$ at position $x$ and time $t$

\footnotetext{
${ }^{1}$ This research was supported by the National Natural Science Foundation of China.
} 
can be described by the following coupled differential equation:

$$
\left\{\begin{array}{l}
\rho y_{t t}(x, t)+E I y_{x x x x}(x, t)=0, \quad 0<x<\ell, \quad t>0 \\
y(0, t)=0 \\
E I y_{x x}(0, t)-I_{m} y_{x t t}(0, t)+u(t)=0 \\
E I y_{x x x}(\ell, t)-M y_{t t}(\ell, t)=0 \\
E I y_{x x}(\ell, t)+J y_{x t t}(\ell, t)=0 .
\end{array}\right.
$$

Let the terminal state trajectory be $x \theta_{d}(t)$, where $\theta_{d}^{\prime \prime}(t)=0$, i.e., the tracked state would be uniform motion or fixed in some direction for the flexible beam. Thus the difference displacement $e(x, t)=y(x, t)-x \theta_{d}(t)$ will satisfy the same equation (1). By this fact, we will, here and throughout this paper, use $y(x, t)$ to represent the error displacement as well as total displacement. It is obvious that the feedback control should make the energy of the dynamic system (1) be decreasing with time.

Let us briefly outline the content of this paper. In section 2, we design a dissipative nonlinear feedback control with angular velocity of motor and show the well-posedness of the closed loop system. It is also shown that the energy in this case will tend to zero as time goes to infinity. Section 3 is devoted to the uniform decay estimate of the closed loop system with the angular acceleration feedback.

\section{Well-posedness of the Problem and the Asymptotic Stability}

We design a nonlinear dissipative feedback control by

$$
u(t)=-\alpha y_{x}(0, t)-f\left(y_{x t}(0, t)\right)
$$

(where $\alpha>0$ is a positive constant) and study the following closed-loop system

$$
\left\{\begin{array}{l}
\rho y_{t t}(x, t)+E I y_{x x x x}(x, t)=0, \quad 0<x<\ell, \quad t>0 \\
y(0, t)=0 \\
\quad E I y_{x x}(0, t)-I_{m} y_{x t t}(0, t)-\alpha y_{x}(0, t)-f\left(y_{x t}(0, t)\right)=0 \\
E I y_{x x x}(\ell, t)-M y_{t t}(\ell, t)=0 \\
E I y_{x x}(\ell, t)+J y_{x t t}(\ell, t)=0
\end{array}\right.
$$

where the feedback function $f$ such that $f \in C^{0}(\mathbb{R})$ is increasing with

$$
f(0)=0 \text { and } s f(s)>0 \text { for } s \neq 0 .
$$

Let $\mathcal{H}_{6}=H_{E}^{2}(0, \ell) \times L^{2}(0, \ell) \times \mathbb{R}^{3}$ be the underlying state space with the inner product

$$
\begin{aligned}
& \left\langle\left(u(x), v(x), a_{1}, a_{2}, a_{3}\right),\left(\bar{u}(x), \bar{v}(x), \bar{a}_{1}, \bar{a}_{2}, \bar{a}_{3}\right)\right\rangle \\
& =\frac{1}{2} \int_{0}^{\ell}\left[E I u^{\prime \prime}(x) \bar{u}^{\prime \prime}(x)+\rho v(x) \bar{v}(x)\right] d x+\frac{1}{2} \alpha u^{\prime}(0) \bar{u}^{\prime}(0)+\frac{1}{2}\left[I_{m} a_{1} \bar{a}_{1}+M a_{2} \bar{a}_{2}+J a_{3} \bar{a}_{3}\right],
\end{aligned}
$$


where $H_{E}^{2}(0, \ell)=\left\{u(x) \mid u \in H^{2}(0, \ell), u(0)=0\right\}$. Define a nonlinear operator $\mathcal{A}: D(\mathcal{A})(\subset \mathcal{H}) \rightarrow \mathcal{H}$ by

$$
\begin{gathered}
\mathcal{A}\left(u(x), v(x), v^{\prime}(0), v(\ell), v^{\prime}(\ell)\right) \\
=\left(-v(x), \frac{E I}{\rho} u^{(4)}(x),-\frac{E I}{I_{m}} u^{\prime \prime}(0)+\frac{\alpha}{I_{m}} u^{\prime}(0)+\frac{1}{I_{m}} f\left(v^{\prime}(0)\right),\right. \\
\left.-\frac{E I}{M} u^{\prime \prime \prime}(\ell), \frac{E I}{J} u^{\prime \prime}(\ell)\right) \\
D(\mathcal{A})=\left\{\left(u(x), v(x), v^{\prime}(0), v(\ell), v^{\prime}(\ell)\right), u \in H^{4}(0, \ell)\right. \\
\left.v \in H^{2}(0, \ell), u(0)=v(0)=0\right\}
\end{gathered}
$$

Then equation (2) can be written as a nonlinear evolution equation on $\mathfrak{H}$ :

$$
\left\{\begin{array}{l}
\frac{d Y(t)}{d t}+\mathcal{A} Y(t)=0 \\
Y(0)=Y_{0}
\end{array}\right.
$$

where $Y(t)=\left(y(x, t), y_{t}(x, t), y_{x t}(0, t), y_{t}(\ell, t), y_{x t}(\ell, t)\right)^{T}$. Notice that the norm of state is just the energy of the system:

$$
\begin{aligned}
E(t)=\| & Y(t) \|^{2} \\
& =\frac{1}{2} \int_{0}^{\ell}\left[\rho y_{t}^{2}+E I y_{x x}^{2}\right] d x+\frac{1}{2} \alpha y_{x}^{2}(0, t)+\frac{1}{2} I_{m} y_{x t}^{2}(0, t)+\frac{1}{2} M y_{t}^{2}(\ell, t)+\frac{1}{2} J y_{x t}^{2}(\ell, t),
\end{aligned}
$$

and formally

$$
\frac{d E(t)}{d t}=-y_{x t}(0, t) f\left(y_{x t}(0, t)\right) \leq 0 .
$$

Lemma 1: Under the assumption (3), the operator $\mathcal{A}$ defined by (4) is maximal monotone on H6 with the domain $D(\mathcal{A})$ that is dense in $\mathcal{H} 6$.

Proof: Let $U, V \in D(\mathcal{A})$, where

$$
\begin{aligned}
U & =\left(u(x), v(x), v^{\prime}(0), v(\ell), v^{\prime}(\ell)\right), \\
V & =\left(\bar{u}(x), \bar{v}(x), \bar{v}^{\prime}(0), \bar{v}(\ell), \bar{v}^{\prime}(\ell)\right) .
\end{aligned}
$$

Then a simple calculation yields

$$
\langle\mathcal{A} U-\mathcal{A} V, U-V\rangle=\frac{1}{2}\left[f\left(v^{\prime}(0)\right)-f\left(\bar{v}^{\prime}(0)\right)\right]\left[v^{\prime}(0)-\bar{v}^{\prime}(0)\right] \geq 0 .
$$

This means that $\mathcal{A}$ is monotone. To prove the maximal monotonicity of $\mathcal{A}$ it is sufficient to prove the range condition (see [1])

$$
\mathscr{B}(I+\mathcal{A})=\mathfrak{H}
$$

i.e., for any given $\left.u_{0}(x), v_{0}(x), a_{1}, a_{2}, a_{3}\right) \in \mathcal{H} \mathcal{G}$, there exists $\left(u(x), v(x), v^{\prime}(0), v(\ell), v^{\prime}(\ell)\right) \in D(\mathcal{A})$ such that $v(x)=u(x)-u_{0}(x)$ and $u$ satisfies 


$$
\left\{\begin{array}{l}
\quad \frac{E I}{\rho} u^{(4)}(x)+u(x)=u_{0}(x)+v_{0}(x), u(0)=0 \\
\left(1+\frac{\alpha}{I_{m}}\right) u^{\prime}(0)-\frac{E I}{I_{m}} u^{\prime \prime}(0)+\frac{1}{I_{m}} f\left(u^{\prime}(0)-u_{0}^{\prime}(0)\right)=a_{1}+u_{0}^{\prime}(0) \\
u(\ell)-\frac{E I}{M} u^{\prime \prime \prime}(\ell)=a_{2}+u_{0}(\ell) \\
u^{\prime}(\ell)+\frac{E I}{J} u^{\prime \prime}(\ell)=a_{3}+u_{0}^{\prime}(\ell)
\end{array}\right.
$$

If $u(x) \in H^{4}(0, \ell)$ is a solution of (7), then multiplying by $\rho \phi(x) \in H_{E}^{2}(0, \ell)$ both sides of the first equation of (7) and integrating from 0 to $\ell$ with respect to $x$, we have

$$
a(u, \phi)-F(\phi)+f\left(u^{\prime}(0)-u_{0}^{\prime}(0)\right) \phi^{\prime}(0)=0, \quad \phi(x) \in H_{E}^{2}(0, \ell)
$$

where the bilinear functional $a(\cdot, \cdot)$ is defined and coercive on $H_{E}^{2}(0, \ell)$ and a linear bounded functional $F(\cdot)$ is defined on $H_{E}^{2}(0, \ell)$ as follows:

$$
\begin{aligned}
a(\psi, \phi) & =\int_{0}^{\ell}\left[E I \psi^{\prime \prime}(x) \phi^{\prime \prime}(x)+\rho \psi(x) \phi(x)\right] d x \\
& +M \psi(\ell) \phi(\ell)+J \psi^{\prime}(\ell) \phi^{\prime}(\ell)+\left(\alpha+I_{m}\right) \psi^{\prime}(0) \phi^{\prime}(0), \quad \forall \psi, \phi \in H_{E}^{2}(0, \ell), \\
F(\psi) & =M\left[a_{2}+u_{0}(\ell)\right] \psi(\ell)+J\left[a_{3}+u_{0}^{\prime}(\ell)\right] \psi^{\prime}(\ell) \\
& +I_{m}\left[a_{1}+u_{0}^{\prime}(0)\right] \psi^{\prime}(0)+\rho \int_{0}^{\ell}\left[u_{0}(x)+v_{0}(x)\right] \psi(x) d x, \quad \forall \psi \in H_{E}^{2}(0, \ell) .
\end{aligned}
$$

On the other hand, let

$$
J(\psi)=\frac{1}{2} a(\psi, \psi)-F(\psi)+\int_{0}^{\psi^{\prime}(0)-u_{0}^{\prime}(0)} f(s) d s, \quad \forall \psi \in H_{E}^{2}(0, \ell)
$$

Since $\int_{0}^{\psi^{\prime}(0)-u_{0}^{\prime}(0)} f(s) d s \geq 0, J(\cdot)$ is convex, coercive and strongly continuous on $H_{E}^{2}(0, \ell)$, there exists a unique function $u \in H_{E}^{2}(0, \ell)$ such that

This means that for all $\phi \in H_{E}^{2}(0, \ell)$,

$$
J(u)=\inf _{\psi \in H_{E}^{2}(0, \ell)} J(\psi) .
$$

$$
a(u, \phi)-F(\phi)+f\left(u^{\prime}(0)-u_{0}^{\prime}(0)\right) \phi^{\prime}(0)=0,
$$

or $u(x) \in H_{E}^{2}(0, \ell)$ satisfies equation (7) in the sense of distribution.

Next, since equation (7) is a regular elliptic boundary value problem, from classical elliptic theory [3],

$$
u \in H^{4}(0, \ell)
$$

Because $u_{0}(x) \in H^{2}(0, \ell)$, we see that $v(x)=u(x)-u_{0}(x) \in H^{2}(0, \ell)$, and

$$
(I+\mathcal{A})\left(u(x), v(x), v^{\prime}(0), v(\ell), v^{\prime}(\ell)\right)=\left(u_{0}(x), v_{0}(x), a_{1}, a_{2}, a_{3}\right) .
$$


So, $\mathscr{B}(I+\mathcal{A})=\mathfrak{H} \mathscr{6}$. Finally, if there is $U \in \mathfrak{H} \mathscr{6}$ such that

$$
\langle U, V\rangle=0, \quad \forall V \in D(\mathcal{A})
$$

Assume that $U=(I+\mathcal{A}) U_{0}$ for some $U_{0} \in \mathcal{H}$ G. Then, $\left\langle U_{0}, U_{0}\right\rangle \leq\left\langle U, U_{0}\right\rangle=0$, which implies that $U_{0}=U=0$. Thus $D(\mathcal{A})$ is dense in $\mathcal{H} 6$.

Since the operator $\mathcal{A}$ is maximal monotone with the dense domain $D(\mathcal{A})$ in the energy space J6, by applying a method developed in [2] to the evolution equation (2), we obtain the following existence result.

Theorem 1: Assume that validity of (3). Then we have

(i) For $Y_{0}=\left(y_{0}(x), y_{1}(x), y_{1}^{\prime}(0), y_{1}(\ell)\right), y_{1}^{\prime}(\ell)^{T} \in D(\mathcal{A})$, equation (2) (and (5)) has a unique strong solution $Y(t)$ with $Y(0)=Y_{0}$, such that

$$
\begin{gathered}
Y(t)=\left(y(x, t), y_{t}(x, t), y_{x t}(0, t), y_{t}(\ell, t), y_{x t}(\ell, t)\right)^{T} \in D(\mathcal{A}), \forall t \geq 0 \\
y(x, t) \in W^{1, \infty}\left(\mathbb{R}^{+} ; H^{2}(0, \ell)\right) \cap L^{\infty}\left(\mathbb{R}^{+} ; H^{4}(0, \ell)\right) \\
\left(y_{x t}(0, t), y_{t}(\ell, t), y_{x t}(\ell, t)\right) \in\left(W^{1, \infty}\left(\mathbb{R}^{+}, \mathbb{R}\right)\right)^{3} \\
\|Y(t)\| \leq\left\|Y_{0}\right\| .
\end{gathered}
$$

(ii) For any initial data $Y_{0}=\left(y_{0}(x), y_{1}(x), a_{1}, a_{2}, a_{3}\right)^{T} \in \mathcal{H}$, equation (2) (and (5)) has a unique weak solution, with

$$
Y(t)=\left(y(x, t), y_{t}(x, t), y_{x t}(0, t), y_{t}(\ell, t), y_{x t}(\ell, t)\right)^{T}=S(t) Y_{0}, \quad \forall t \geq 0,
$$

such that

$$
\begin{gathered}
y(x, t) \in C^{0}\left(\mathbb{R}^{+}, H^{2}(0, \ell)\right) \cap C^{1}\left(\mathbb{R}^{+}, L^{2}(0, \ell)\right), \\
\left(y_{x t}(0, t), y_{t}(\ell, t), y_{x t}(\ell, t)\right) \in\left(C^{1}\left(\mathbb{R}^{+}, \mathbb{R}\right)\right)^{3},
\end{gathered}
$$

where $\{S(t)\}_{t \geq 0}$ denotes the strongly continuous semigroup of contractions on $\mathfrak{H} 6$ generated by maximal monotone operator $\mathcal{A}$.

Lemma 2: The following holds true:

$$
0 \in \mathscr{R}(\mathcal{A}),(I+\mathcal{A})^{-1} \text { is compact. }
$$

Proof: $0 \in \mathscr{R}(\mathcal{A})$ immediately follows from the definition. Thus, we only consider the second condition. Let $\left\{V_{n}\right\} \subseteq \mathfrak{H},\left\|V_{n}\right\| \leq C$, be a bounded series and let $\left\{U_{n}\right\}$ satisfy $(I+\mathcal{A}) U_{n}=V_{n}$. Then, by the monotonicity of $\mathcal{A},\left\|A U_{n}\right\| \leq\left\|V_{n}\right\| \leq C$ and $\left\|U_{n}\right\| \leq\left\|V_{n}\right\| \leq C$. These imply that

$$
\left\|u_{n}\right\|_{H^{4}} \leq C_{1},\left\|v_{n}\right\|_{H^{2}} \leq C_{1}, \quad\left|v_{n}^{\prime}(0)\right| \leq C_{1},\left|v_{n}(\ell)\right| \leq C_{1},\left|v_{n}^{\prime}(\ell)\right| \leq C_{1}
$$

for some uniform constant $C_{1}$, provided that

$$
U_{n}=\left(u_{n}(x), v_{n}(x), v_{n}^{\prime}(0), v_{n}(\ell), v_{n}^{\prime}(\ell)\right)^{T} .
$$

By the Sobolev embedding theorem, there is a subsequence of $U_{n}$, still indexed by $n$ for notational 
simplicity, and $U_{0} \in H^{2}(0, \ell) \times L^{2}(0, \ell) \times \mathbb{R}^{2}$, such that

$$
U_{n} \rightarrow U_{0} \text { in the topology of } \mathfrak{H} \text {. }
$$

$u_{0}(0)=0$ is hence an obvious fact. The proof is complete.

Lemma 3: Let $S(t)$ be the semigroup defined by Theorem 1. If for some $Y_{0}=\left(y_{0}(x), y_{1}(x)\right.$, $\left.y_{1}^{\prime}(0), y_{1}(\ell), y^{\prime}(\ell)\right)^{T} \in D(\mathcal{A})$,

$$
\frac{d E(t)}{d t}=\frac{d}{d t} \| S(t) Y_{0} \mid \equiv 0, \quad t \geq 0
$$

then $Y_{0}=0$.

Proof: Let $Y(t)=\left(y(x, t), y_{t}(x, t), y_{x t}(0, t), y_{t}(\ell, t), y_{x t}(\ell, t)\right)^{T}$ be a solution of equation (2) with the initial condition $Y(0)=Y_{0}$. By (6) and assumption (3), $\frac{d}{d t}\left\|S(t) Y_{0}\right\| \equiv 0$ means that

$$
\left\{\begin{array}{l}
\rho y_{t t}(x, t)+E I y_{x x x x}(x, t)=0, \quad 0<x<\ell, \quad t>0 \\
y(0, t)=y_{x t}(0, t)=0 \\
E I y_{x x}(0, t)-\alpha y_{x}(0, t)=0 \\
E I y_{x x x}(\ell, t)-M y_{t t}(\ell, t)=0 \\
E I y_{x x}(\ell, t)+J y_{x t t}(\ell, t)=0 .
\end{array}\right.
$$

Multiplying by $x$ on both sides of the first equation of (8) and integrating it from 0 to $\ell$ with respect to $x$, we have

$$
\rho \int_{0}^{\ell} z y_{t t}(z, t) d z+M \ell y_{t t}(\ell, t)+J y_{x t t}(\ell, t)+E I y_{x x}(0, t)=0 .
$$

Then, integrating (9) from 0 to $T>0$ with respect to $t$ one gets

$$
\begin{aligned}
& E I \int_{0}^{T} y_{x x}(0, t) d t \\
& \quad=\rho \int_{0}^{\ell} z\left[y_{t}(z, T)-y_{t}(z, 0)\right] d z-M \ell\left[y_{t}(\ell, T)-y_{t}(\ell, 0)\right]-J\left[y_{x t}(\ell, T)-y_{x t}(\ell, 0)\right],
\end{aligned}
$$

which, along with the fact that $E(t)=E(0)$, imply that

$$
\left|\int_{0}^{T} y_{x x}(0, t) d t\right| \leq \text { Const. }
$$

Furthermore, noticing that $y_{x}(0, t)$ is constant, from the boundary condition at $x=0$, we obtain

which implies that

$$
\alpha y_{x}(0, t) T=\int_{0}^{T} E I y_{x x}(0, t) d t, \quad \forall T>0,
$$

$$
\alpha y_{x}(0, t)=y_{x x}(0, t)=y_{x t t}(0, t)=0, \quad \forall t \geq 0 .
$$

Next, multiplying by $y(x, t)$ and $2(x-\ell-\epsilon) y_{x}(x, t)$ both sides of the first equation of (8) and 
then integrating with respect to $x$ and $t$, respectively, where $\epsilon>0$ is constant (to be determined), we obtain

$$
\begin{aligned}
& -\int_{0}^{T} \int_{0}^{\ell}\left[\rho y_{t t}(x, t) y(t, x)+E I y_{x x x x}(x, t) y(x, t)\right] d x d t \\
& \quad=-E I \int_{0}^{T} \int_{0}^{\ell} y_{x x}^{2} d x d t+\rho \int_{0}^{T} \int_{0}^{\ell} y_{t}^{2} d x d t+M \int_{0}^{T} y_{t}^{2}(\ell, t) d t \\
& \quad+J \int_{0}^{T} y_{x t}^{2}(\ell, t) d t-\left.M y_{t} y(\ell, t)\right|_{0} ^{T}+\left.J y_{x t} y_{x}(\ell, t)\right|_{0} ^{T}-\left.\rho \int_{0}^{\ell} y_{t} y(x, t)\right|_{0} ^{T} d x=0,
\end{aligned}
$$

and

$$
\begin{gathered}
2 \int_{0}^{T} \int_{0}^{\ell}(x-\ell-\epsilon) y_{x}(x, t)\left[\rho y_{t t}(x, t)+E I y_{x x x x}(x, t)\right] d x d t \\
=\int_{0}^{T} \int_{0}^{\ell}\left[\rho y_{t}^{2}+3 E I y_{x x}^{2}\right] d x d t+\rho \epsilon \int_{0}^{T} y_{t}^{2}(\ell, t) d t+2 M \epsilon \int_{0}^{T} y_{x t}(\ell, t) y_{t}(\ell, t) d t \\
-2 J \int_{0}^{T} y_{x t}^{2}(\ell, t) d t+\left.2 \rho \int_{0}^{\ell}(x-\ell-\epsilon) y_{t} y_{x}\right|_{0} ^{T} d x-\left.2 M \epsilon y_{x}(\ell, t) y_{t}(\ell, t)\right|_{0} ^{T} \\
+\left.2 J y_{x}(\ell, t) y_{x t}(\ell, t)\right|_{0} ^{T}+E I \epsilon \int_{0}^{T} y_{x x}^{2}(\ell, t) d t=0 .
\end{gathered}
$$

Computing $(2+\delta) \times(10)+(11)$ for $\delta=2 M / J \epsilon$, we have

$$
\begin{aligned}
& \int_{0}^{T} \int_{0}^{\ell}\left[\rho(3+\delta) y_{t}^{2}+E I(1-\delta) y_{x x}^{2}\right] d x d t+[(2+\delta) M+\rho \epsilon] \int_{0}^{T} y_{t}^{2}(\ell, t) d t \\
& \quad+J \delta \int_{0}^{T} y_{x t}^{2}(\ell, t) d t+2 M \epsilon \int y_{x t}(\ell, t) y_{t}(\ell, t) d t+E I \epsilon \int_{0}^{T} y_{x x}^{2}(\ell, t) d t+C(t)=0,
\end{aligned}
$$

where

$$
\begin{aligned}
& C(t)=(2+\delta)\left[-\left.M y_{t}(\ell, t) y(\ell, t)\right|_{0} ^{T}+\left.J y_{x t}(\ell, t) y_{x}(\ell, t)\right|_{0} ^{T}+\left.\rho \int_{0}^{\ell} y y_{t}\right|_{0} ^{T} d x\right] \\
& -\left.2 \rho \int_{0}^{\ell}(x-\ell-\epsilon) y_{t} y_{x}\right|_{0} ^{T} d x-\left.2 M \epsilon y_{x}(\ell, t) y_{t}(\ell, t)\right|_{0} ^{T}+\left.2 J y_{x}(\ell, t) y_{x t}(\ell, t)\right|_{0} ^{T} .
\end{aligned}
$$

Obviously, $|C(t)| \leq$ Const $\cdot E(t)$. So,

$$
\int_{0}^{T} \int_{0}^{\ell}\left[\rho(3+\delta) y_{t}^{2}+E I(1-\delta) y_{x x}\right] d x d t
$$




$$
\begin{aligned}
& \leq-[(2+\delta) M+\rho \epsilon-M \epsilon] \int_{0}^{T} y_{t}^{2}(\ell, t) d t-[J \delta-M \epsilon] \int_{0}^{T} y_{x t}^{2}(\ell, t) d t-C(t) . \\
& \leq-(2-\epsilon) M \int_{0}^{T} y_{t}^{2}(\ell, t) d t-M \epsilon \int_{0}^{T} y_{x t}^{2}(\ell, t) d t-C(t) .
\end{aligned}
$$

Taking $0<\epsilon<\min \{1, J /(2 M)\},(12)$ implies that

$$
\int_{0}^{T} E(t) d t \leq \text { Const. }
$$

Thus $E(t) \equiv 0$ by the arbitrariness of $T$ and $E(t)=E(0)$. The proof is complete.

By Theorem 1, Lemmas 2 and 3, and using LaSalle's invariance principle [1], we have immediately:

Theorem 2: Let $E(t)$ be the energy of the hybrid system (2). Then,

$$
\lim _{t \rightarrow \infty} E(t)=0 .
$$

\section{The Uniform Decay}

To get the uniform decay, we design, in this section, a boundary feedback control as

$$
u(t)=I_{m} y_{x t t}(0, t)-\alpha y_{x}(0, t)-f\left(y_{x t}(0, t)\right) .
$$

Then the closed loop system would be

$$
\left\{\begin{array}{l}
\rho y_{t t}(x, t)+E I y_{x x x x}(x, t)=0, \quad 0<x<\ell, \quad t>0 \\
y(0, t)=0 \\
E I y_{x x}(0, t)-\alpha y_{x}(0, t)-f\left(y_{x t}(0, t)\right)=0, \\
E I y_{x x x}(\ell, t)-M y_{t t}(\ell, t)=0, \\
E I y_{x x}(\ell, t)+J y_{x t t}(\ell, t)=0 .
\end{array}\right.
$$

In this case, the energy takes the form

$$
E(t)=\frac{1}{2} \int_{0}^{\ell}\left[\rho y_{t}^{2}+E I y_{x x}^{2}\right] d x+\frac{1}{2} \alpha y_{x}^{2}(0, t)+\frac{1}{2} M y_{t}^{2}(\ell, t)+\frac{1}{2} J y_{x t}^{2}(\ell, t) .
$$

Consequently, the underlying state space becomes $\mathfrak{H}_{6}=H_{E}^{2}(0, \ell) \times L^{2}(0, \ell) \times \mathbb{R}^{2}$ with the inner product

$$
\left\langle\left(u(x), v(x), a_{1}, a_{2}\right),\left(\bar{u}(x), \bar{v}(x), \bar{a}_{1}, \bar{a}_{2}\right)\right\rangle
$$




$$
=\frac{1}{2} \int_{0}^{\ell}\left[E I u^{\prime \prime}(x) \bar{u}^{\prime \prime}(x)+\rho v(x) \bar{v}(x)\right] d x+\frac{1}{2} \alpha u^{\prime}(0) \bar{u}^{\prime}(0)+\frac{1}{2}\left[M a_{1} \bar{a}_{1}+J a_{2} \bar{a}_{2}\right],
$$

where $H_{E}^{2}(0, \ell)$ is defined in section 2. Equation (14) can also be written as a nonlinear evolution equation in 36 :

$$
\left\{\begin{array}{l}
\frac{d Y(t)}{d t}+\mathcal{A} Y(t)=0 \\
Y(0)=Y_{0}
\end{array}\right.
$$

but instead of $(4)$, here $\mathcal{A}: D(\mathcal{A})(\subset \mathfrak{H}) \rightarrow \mathfrak{H}$ is defined by

$$
\begin{aligned}
& \mathcal{A}\left(u(x), v(x), v(\ell), v^{\prime}(\ell)\right)=\left(-v(x), \frac{E I}{\rho} u^{(4)}(x),-\frac{E I}{M} u^{\prime \prime \prime}(\ell), \frac{E I}{J} u^{\prime \prime}(\ell)\right) \\
& D(\mathcal{A})=\left\{\left(u(x), v(x), v(\ell), v^{\prime}(\ell)\right), u \in H^{4}(0, \ell),\right. \\
& \left.v \in H^{2}(0, \ell), u(0)=v(0)=E I u^{\prime \prime}(0)-\alpha u^{\prime}(0)-f\left(v^{\prime}(0)\right)=0\right\}
\end{aligned}
$$

and state variable $Y(t)=\left(y(x, t), y_{t}(x, t), y_{t}(\ell, t), y_{x t}(\ell, t)\right)^{T}$.

Following the same line as that of section 2, we also have

Theorem 3: The operator $\mathcal{A}$ defined by (17) is maximal monotone with the dense domain in the space $J 6$ and hence generates an asymptotically stable nonlinear semigroup of contractions on J6.

In the sequel, we always assume that the initial data belongs to the domain of operator $\mathcal{A}$ and hence the solution of equation (14) has the regularity properties expressed by (ii) of Theorem 1. Let $\delta>0$ and $\phi(x)=a x-a \ell-1$, where $a$ is a constant to be determined. Define

$$
\begin{aligned}
& \beta(t)=2 \int_{0}^{\ell} \phi(x) y_{t}(x, t) y_{x}(x, t) d x \\
& -2 \phi(0) y_{x}(0, t)\left[\int_{0}^{\ell} y_{t} d x+\frac{\alpha}{E I} \int_{0}^{\ell} x y_{t} d x+\frac{M}{\rho}\left(1+\frac{\alpha \ell}{E I}\right) y_{t}(\ell, t)+\frac{\alpha J}{E I \rho} y_{x t}(\ell, t)\right] \\
& +\frac{2}{\rho} y_{x}(\ell, t)\left[M \phi(\ell) y_{t}(\ell, t)+J \phi^{\prime}(\ell) y_{x t}(\ell, t)\right] \\
& +(2+\delta) \phi^{\prime}\left[\int_{0}^{\ell} y_{t} y d x+\frac{M}{\rho} y(\ell, t) y_{t}(\ell, t)+\frac{J}{\rho} y_{x}(\ell, t) y_{x t}(\ell, t)\right]
\end{aligned}
$$

Lemma 4: Let $\beta(t)$ be defined by (18). Then there exist a $\delta>0$ and positive constants $C_{0}$, $C_{1}, C_{2}$, and $C_{3}$ such that

$$
\begin{aligned}
& |\beta(t)| \leq C_{0} E(t), \\
& B^{\prime}(t) \leq-C_{1} E(t)+C_{2} y_{x t}^{2}(0, t)+C_{3} f^{2}\left(y_{x t}(0, t)\right) .
\end{aligned}
$$

Proof: By the defined form of $\beta(t)$ and Sobolev's embedding theorem, we can always find a constant $C_{0}$ such that $|\beta(t)| \leq C_{0} E(t)$ once the constants $a$ and $\delta$ are determined. To prove the second condition, we find the derivative $\beta^{\prime}(t)$ directly. 
Let

$$
\begin{aligned}
& \beta_{1}(t)=2 \int_{0}^{\ell} \phi(x) y_{t}(x, t) y_{x}(x, t) d x \\
& \beta_{2}(t)=-2 \phi(0) y_{x}(0, t)\left[\int_{0}^{\ell} y_{t} d x+\frac{\alpha}{E I} \int_{0}^{\ell} x y_{t} d x+\frac{M}{\rho}\left(1+\frac{\alpha \ell}{E I}\right) y_{t}(\ell, t)+\frac{\alpha J}{E I \rho} y_{x t}(\ell, t)\right] \\
& \beta_{3}(t)=\frac{2}{\rho} y_{x}(\ell, t)\left[M \phi(\ell) y_{t}(\ell, t)+J \phi^{\prime}(\ell) y_{x t}(\ell, t)\right] \\
& \beta_{4}(t)=(2+\delta) \phi^{\prime}\left[\int_{0}^{\ell} y_{t} y d x+\frac{M}{\rho} y(\ell, t) y_{t}(\ell, t)+\frac{J}{\rho} y_{x}(\ell, t) y_{x t}(\ell, t)\right] .
\end{aligned}
$$

After a tedious but very straightforward calculation we find that

$$
\begin{aligned}
\beta_{1}^{\prime}(t)= & -\int_{0}^{\ell} \phi^{\prime} y_{t}^{2} d x-\frac{3 E I}{\rho} \int_{0}^{\ell} \phi^{\prime} y_{x x}^{2} d x+\phi(\ell) y_{t}^{2}(\ell, t) \\
& -\frac{2 E I}{\rho}\left[\left.\phi y_{x x x} y_{x}\right|_{0} ^{\ell}-\phi^{\prime}(\ell) y_{x}(\ell, t) y_{x x}(\ell, t)+\frac{1}{2} \phi(\ell) y_{x x}^{2}(\ell, t)\right] \\
& -\frac{2 \alpha}{\rho} \phi(0) y_{x x}(0, t) y_{x}(0, t)+\frac{\alpha}{\rho}\left(\frac{\alpha \phi(0)}{E I}-2 \phi^{\prime}(0) y_{x}^{2}(0, t)\right. \\
& +\frac{2 \phi^{\prime}(0)}{\rho} y_{x}(0, t) f\left(y_{x t}(0, t)\right)-\frac{\phi(0)}{\rho E I} f^{2}\left(y_{x t}(0, t)\right) \\
\beta_{2}^{\prime}(t)= & -2 \phi(0) y_{x t}(0, t)\left[\int_{0}^{\ell} y_{t} d x+\frac{2}{E I} \int_{0}^{\ell} x y_{t} d x+\frac{M}{\rho}\left(1+\frac{\alpha \ell}{E I}\right) y_{t}(\ell, t)+\frac{\alpha J}{E I \rho} y_{x t}(\ell, t)\right] \\
& -2 \phi(0) y_{x}(0, t)\left[-\frac{E I}{\rho} y_{x x x}(0, t)-\frac{\alpha}{\rho} y_{x x}(0, t)\right] \\
\beta_{3}^{\prime}(t)= & \frac{2}{\rho} y_{x t}(\ell, t)\left[M \phi(\ell) y_{t}(\ell, t)+J \phi^{\prime}(\ell) y_{x t}(\ell, t)\right] \\
& +\frac{2}{\rho} y_{x}(\ell, t)\left[M \phi(\ell) y_{t t}(\ell, t)+J \phi^{\prime}(\ell) y_{x t t}(\ell, t)\right] \\
& \left.+\frac{J}{\rho} y_{x t}^{2}(\ell, t)-\frac{\alpha}{\rho} y_{x}^{2}(0, t)-\frac{1}{\rho} y_{x}^{2}(0, t) f\left(y_{x t}(0, t)\right)\right] \\
\beta_{4}^{\prime}(t)= & -(2+\delta) \phi^{\prime}\left[\int_{0}^{\ell} y_{t}^{2} d x-\frac{E I}{\rho} \int_{0}^{\ell} y_{x x}^{2} d x+\frac{M}{\rho} y_{t}^{2}(\ell, t)\right. \\
& -(\ell) \\
&
\end{aligned}
$$

Therefore,

$$
\begin{aligned}
\beta^{\prime}(t) & =\beta_{1}^{\prime}(t)+\beta_{2}^{\prime}(t)+\beta_{3}^{\prime}(t)+\beta_{4}^{\prime}(t) \\
& =-a \int_{0}^{\ell}\left[(3+\delta) y_{t}^{2}+(1-\delta) \frac{E I}{\rho} y_{x x}^{2}\right] d x-\frac{\alpha}{\rho}\left[\left(\frac{\alpha \ell}{E I}-\delta\right) a+\frac{\alpha}{E I}\right] y_{x}^{2}(0, t)
\end{aligned}
$$




$$
\begin{aligned}
& -\left[1+(2+\alpha) \frac{M a}{\rho}\right] y_{t}^{2}(\ell, t)-\frac{J \delta a}{\rho} y_{x t}^{2}(\ell, t) \\
& -\frac{E I}{\rho} y_{x x}^{2}(\ell, t)-\frac{2 M}{\rho} y_{x t}(\ell, t) y_{t}(\ell, t)-\beta_{0}(t) \\
\leq & -a \int_{0}^{\ell}\left[(3+\delta) y_{t}^{2}+(1-\delta) \frac{E I}{\rho} y_{x x}^{2}\right] d x-\frac{\alpha}{\rho}\left[\left(\frac{\alpha \ell}{E I}-\delta\right) a+\frac{\alpha}{E I}\right] y_{x}^{2}(0, t) \\
- & \left(1+\frac{M}{\rho}\left[(2+\delta) a-\frac{1}{\theta}\right]\right) y_{t}^{2}(\ell, t)-\frac{1}{\rho}(\delta J a-M \theta) y_{x t}^{2}(\ell, t)-\beta_{0}(t),
\end{aligned}
$$

where $\theta>0$ is an arbitrary constant and

$$
\begin{aligned}
\beta_{0}(t)= & 2 \phi(0) y_{x t}(0, t)\left[\int_{0}^{\ell} y_{t} d x+\frac{\alpha}{E I} \int_{0}^{\ell} x y_{t} d x+\frac{M}{\rho}\left(1+\frac{\alpha \ell}{E I}\right) y_{t}(\ell, t)+\frac{\alpha J}{E I \rho} y_{x t}(\ell, t)\right] \\
& +\frac{\phi(0)}{E I \rho} f^{2}\left(y_{x t}(0, t)\right)-\frac{\phi^{\prime}(0) \delta}{E I \rho} y_{x}(0, t) f\left(y_{x t}(0, t)\right) .
\end{aligned}
$$

Take

$$
\delta=\min \left\{1 / 2, \frac{\alpha \ell}{2 E I}\right\}, a=\frac{M^{2}+\rho}{\rho J \delta}, \theta=\frac{M}{\rho} .
$$

With these defined constants,

$$
\begin{aligned}
& 1-\delta \geq 1 / 2, \quad\left(\frac{\alpha \ell}{E I}-\delta\right) a+\frac{\alpha}{E I} \geq \frac{\alpha}{E I}, \delta J a-M \theta=1 \\
& 1-\frac{M}{\rho \theta}+(2+\delta) \frac{M a}{\rho}=\frac{(2+\delta) M\left(\rho+M^{2}\right)}{\rho^{2} J \delta}=\mu>0
\end{aligned}
$$

and hence

$$
\beta^{\prime}(t) \leq-a \int_{0}^{\ell}\left[3 y_{t}^{2}+\frac{E I}{2 \rho} y_{x x}^{2}\right] d t-\frac{\alpha^{2}}{\rho E I} y_{x}^{2}(0, t)-\mu y_{t}^{2}(\ell, t)-\frac{1}{\rho} y_{x t}^{2}(\ell, t)-\beta_{0}(t) .
$$

Since $E(t) \leq E(0)$, it is easily seen from (21) and the definition of $\beta_{0}(t)$ that there exist positive constants $C_{1}, C_{2}$, and $C_{3}$ such that

$$
\beta^{\prime}(t) \leq-C_{1} E(t)+C_{2} y_{x t}^{2}(0, t)+C_{3} f^{2}\left(y_{x t}(0, t)\right) .
$$

The proof is complete.

Along the same line as that of [4], we have the following uniform decay estimate as that of [4] made for the hybrid string equation.

Theorem 4: Let (3) hold true. Let y be the solution of equation (14) with initial data being in $D(\mathcal{A})$.

(i) If there exist positive constants $L_{1}$ and $L_{2}$ such that

$$
L_{1}|s| \leq|f(s)| \leq L_{2}|s|, \quad \forall s \in \mathbb{R}
$$

then, given any $K>1$, there exists a constant $\omega>0$ such that

$$
E(t) \leq K E(0) \exp (-\omega t), \quad \forall t \geq 0 .
$$


(ii) If there exist positive constants $L_{1}, L_{2}$ and $p>1$ such that

$$
L_{1} \min \left\{|s|,|s|^{p}\right\} \leq|f(s)| \leq L_{2}|s|, \forall s \in \mathbb{R},
$$

then, given any $K>1$, there exists a constant $\omega>0$ depending continuously on $E(0)$ such that

$$
E(t) \leq K E(0)(1+\omega t)^{-2 /(p-1)}, \quad \forall t \geq 0 .
$$

(iii) If there exist positive constants $L_{1}$ and $L_{2}$ and $0<p<1$ such that

$$
L_{1}|s| \leq|f(s)| \leq L_{2} \max \left\{|s|,|s|^{p}\right\}, \forall s \in \mathbb{R}
$$

then, given any $K>1$, there exists a constant $\omega>0$ depending continuously on $E(0)$ such that

$$
E(t) \leq K E(0)(1+\omega t)^{-2 p /(p-1)}, \forall t \geq 0 .
$$

\section{References}

[1] Dafermos, C.M. and Slemrod, M., Asymptotic behavior of nonlinear contraction semi groups, J. Func. Anal. 13 (1973), 97-106.

[2] Haraux, A., Semilinear hyperbolic problems in bounded domain, In: Math Report, ed. by J. Dieudonne, vol. 3, Harwood Academic Publishers, Gordon and Breach 1987.

[3] Lions, J.L. and Magenenes, E., Non-Homogeneous Boundary Problems, North Holland 1971.

[4] Rao, B.P., Decay estimates of solution for a hybrid system of flexible structures, Euro. J. Appl. Math. 4 (1993), 303-319. 


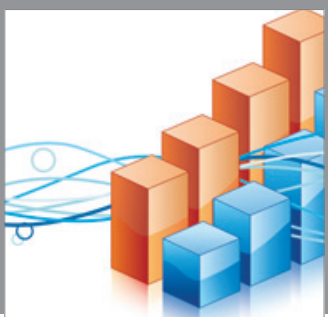

Advances in

Operations Research

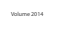

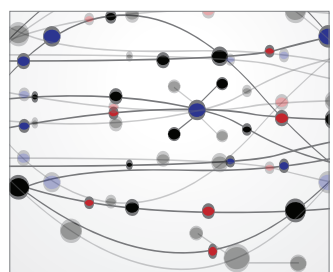

\section{The Scientific} World Journal
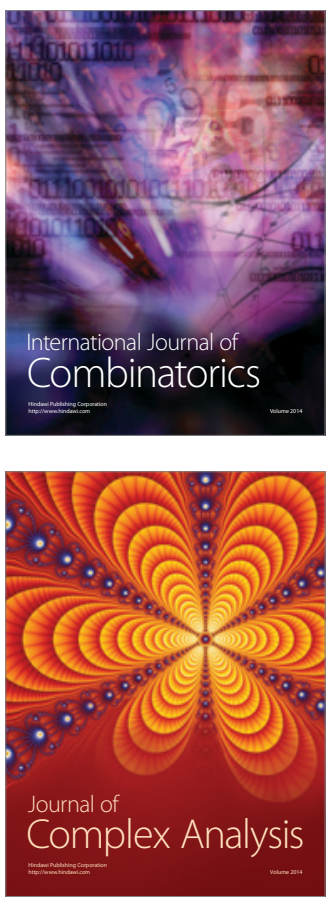

International Journal of

Mathematics and

Mathematical

Sciences
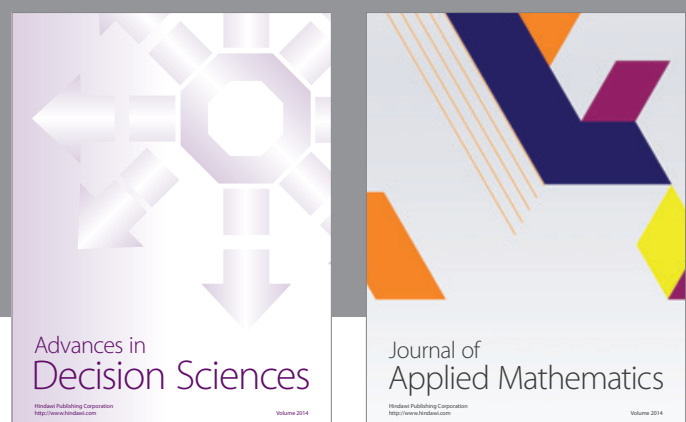

Journal of

Applied Mathematics
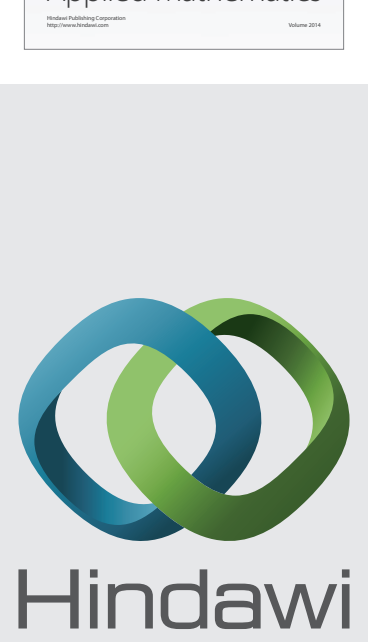

Submit your manuscripts at http://www.hindawi.com
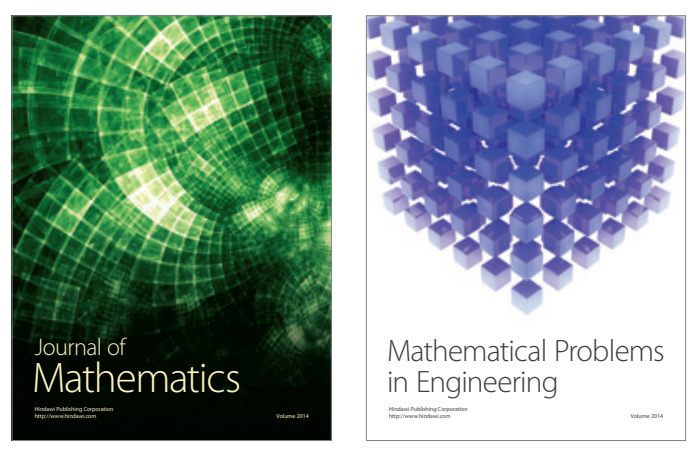

Mathematical Problems in Engineering
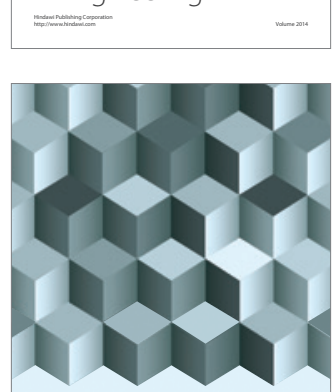

Journal of

Function Spaces
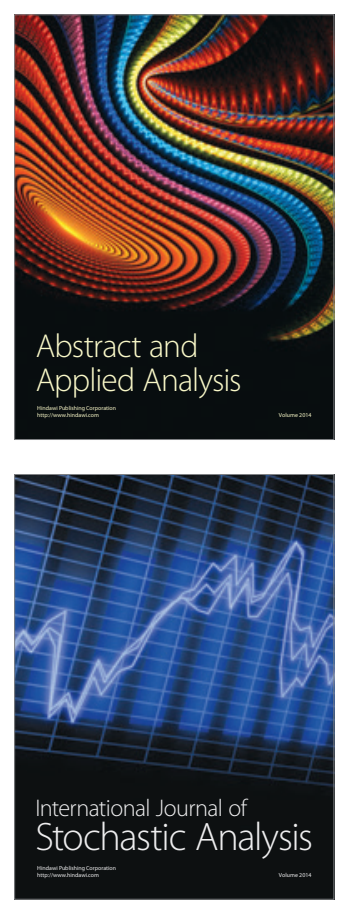

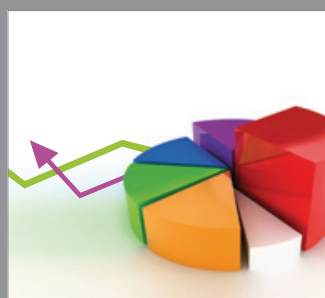

ournal of

Probability and Statistics

Promensencen
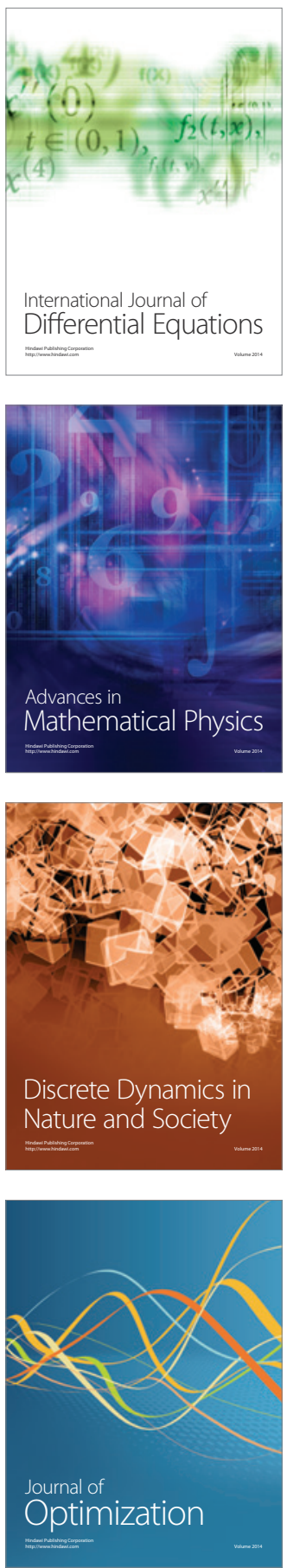